\title{
Rheology and structure of polydisperse three-dimensional packings of spheres
}

\author{
David Cantor, ${ }^{1,2, *}$ Emilien Azéma, ${ }^{1, \dagger}$ Philippe Sornay, ${ }^{2, \ddagger}$ and Farhang Radjai ${ }^{1, \S}$ \\ ${ }^{1}$ LMGC, Univ. Montpellier, CNRS, Montpellier, France \\ ${ }^{2} C E A, D E N$, DEC, SA3E, LCU, F-13108 Saint Paul lez Durance, France
}

(Received 18 April 2018; revised manuscript received 3 September 2018; published 30 November 2018)

\begin{abstract}
We use three-dimensional contact dynamics simulations to analyze the rheology of polydisperse packings of spherical particles subjected to simple shear. The macroscopic and microstructural properties of several packings are analyzed as a function of their size span (from nearly monodisperse to highly polydisperse). Consistently with previous two-dimensional simulations, we find that the shear strength is independent of the size span despite the increase of packing fraction with size polydispersity. At the grain scale, we analyze the particle connectivity, force transmission, and the corresponding anisotropies of the contact and force networks. We show that force distributions become increasingly broader as the size span increases. In particular, stronger forces are captured by large particles, which are also better connected creating the so-called granular backbone. Throughout this backbone friction mobilization is rare and compressive forces control the stability of such structure. In return, small particles create an important population of rattlers discarded of the strength and granular structure analysis. As a consequence, the contact anisotropy declines with size span, whereas the force and branch anisotropies increase. These microstructural compensations allow us to explain the independence of the shear strength from particle size polydispersity.
\end{abstract}

DOI: 10.1103/PhysRevE.98.052910

\section{INTRODUCTION}

Granular materials are commonly found as mixtures of particles of varying sizes. In fault gouges, for example, the longterm shearing between rock masses leads to their wearing and thus the production of particles with a broad size distribution $[1,2]$. In the industry of concrete design and manufacture, this phenomenon is of huge interest as the interactions between pebbles, sand, and cement powder affect the workability of the paste and determine the final strength of the mixture after its setting $[3,4]$. This polydispersity in particle size is thus a major aspect of granular rheology but still poorly investigated from a fundamental perspective.

Indeed, most investigations of the effect of polydispersity have mainly focused on the packing fraction and space filling properties [5-9]. One of the oldest empirical studies on this subject was undertaken by Fuller and Thompson [10] at the beginning of the 20th century in the frame of optimal mixing of cement and aggregates. These authors found that the densest packing is obtained for particle size distributions described by a power law $P(d) \propto d^{0.5}$. Later, a variety of analytical and approximative methods were developed with the aim of predicting the packing fraction of samples composed of monosized [11,12] and polysized [13-19] particles with different particle size distributions. The cases of bidisperse [20-23] and power-law size distributions [24-27] have also been of special interest.

\footnotetext{
*david.cantor@umontpellier.fr

†emilien.azema@umontpellier.fr

†philippe.sornay@cea.fr

§franck.radjai@umontpellier.fr
}

For example, considering a particle size distribution described by a cumulative $\beta$ function, Voivret et al. [7] showed that the densest packing is obtained for a uniform distribution of particle volumes at zero friction, a result that is slightly different from that of Fuller and Thompson. It was only recently that the original work of Fuller and Thompson was confirmed numerically with two-dimensional packings of frictionless disks, by means of the discrete element method [28]. More results on the space-filling properties and the elastic properties of assemblies of grains under uniaxial compression tests were recently analyzed as well by Wiącek et al. [29,30] using 3D-molecular dynamics simulations.

Although the packing properties of polydisperse granular materials have been extensively explored in the past, few studies are dedicated to their quasistatic shearing behavior. There are several issues in dealing with the mechanical behavior of polydisperse granular samples. In general, polydisperse packings need a large number of particles allowing for statistically representative results and, hence, more computational effort is needed for sample preparation and simulations. A strategy for studying polydisperse sheared packings is to analyze simpler systems, e.g., two-dimensional packings composed of circular grains. Following this approach, Voivret et al. [31] found out the counterintuitive phenomenon that the shear strength is independent of grain size span. This finding was later extended to other particle size distributions [32] as well as for noncircular particles [9]. The origin of this property was traced back to the packing microstructure and force networks using a partition of the stress tensor.

In this paper, we investigate the effect of the size span on the shear behavior of three-dimensional packing composed of spherical particles. Our samples present different particle size spans varying from nearly monodisperse to very 
polydisperse with a uniform particle size distribution by volume fractions.

The transition from two-dimensional (2D) to threedimensional (3D) modeling is not trivial and involves several numerical challenges as well as it increases the complexity of the physical analysis. In particular, in 3D samples the pores interconnectivity allows the mobility of small particles and promotes the rearrangement. This impacts the granular texture and the force transmission as well. Besides, the time step discretization has to be fine enough to properly resolve the interactions between particles of the largest and smallest sizes.

Different packings are analyzed in the quasistatic steady state in terms of their shear strength, packing fraction, connectivity, and fabric and force anisotropies as functions of the size span. In Sec. II, we introduce the numerical approach, system characteristics, and loading parameters. In Sec. III, we focus on the dependence of the shear strength and packing fraction with respect to the size span. In Sec. IV, we analyze the granular microstructure and its relation with shear strength. Finally, we summarize our findings and sketch potential perspectives for this work.

\section{NUMERICAL PROCEDURES}

The simulations were carried out by means of the contact dynamics method (CDM) [33-35]. This method is a discrete element approach for the simulation of perfectly rigid particles. In contrast to the molecular dynamics method, in the $\mathrm{CDM}$ the contact laws are expressed in terms of mutual exclusions and solid friction between particles without introducing elastic regularization or viscous damping. Moreover, the implicit time integration scheme used in this method ensures unconditional numerical stability of calculations. The contact dynamics method has been extensively employed for the simulation of granular materials in two and three dimensions with particles of different shapes [32,36-41].

For the simulations analyzed in this paper, we used a uniform distribution of particle volumes, so that the number of particles belonging to a class of diameter $d$ is proportional to $d^{-3}$. This size distribution leads to high packing fractions and corresponds to a "well-graded" sample according to classical soil mechanics classifications [42]. The particle size span is generally defined by considering the classes of largest and smallest particles in the particle size distribution. We characterize the particle size span $S$ in our simulations in terms of the minimum and maximum particle diameter $\left(d_{\min }\right.$ and $d_{\max }$, respectively) as

$$
S=\frac{d_{\max }-d_{\min }}{d_{\max }+d_{\min }} .
$$

The case $S=0$ corresponds to a monodisperse packing whereas $S=1$ corresponds to an "infinite" polydispersity. In this work, $S$ was systematically varied from 0 to 0.8 by steps of 0.1 .

For each value of $S, 20000$ spheres were geometrically placed into a cubic box by means of a sequential layer-bylayer deposition [7]. Figure 1 displays three examples for $S=\{0.0,0.4,0.8\}$.

The samples were compressed isotropically by applying constant stresses to the six walls of the box. During the

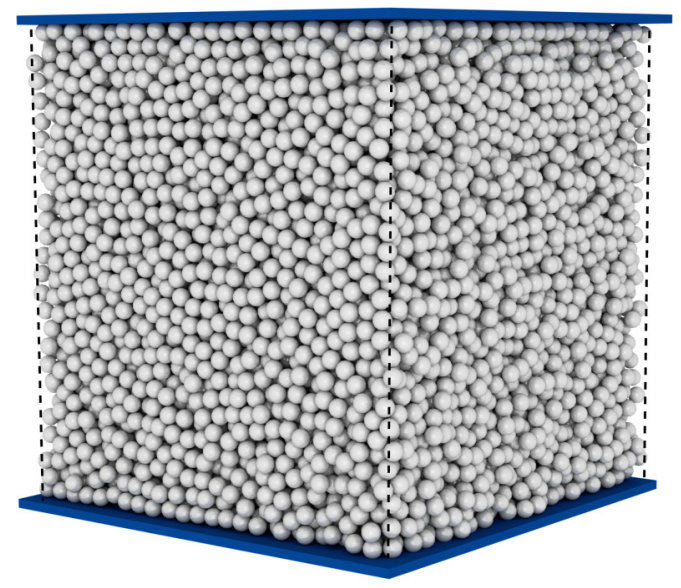

(a)

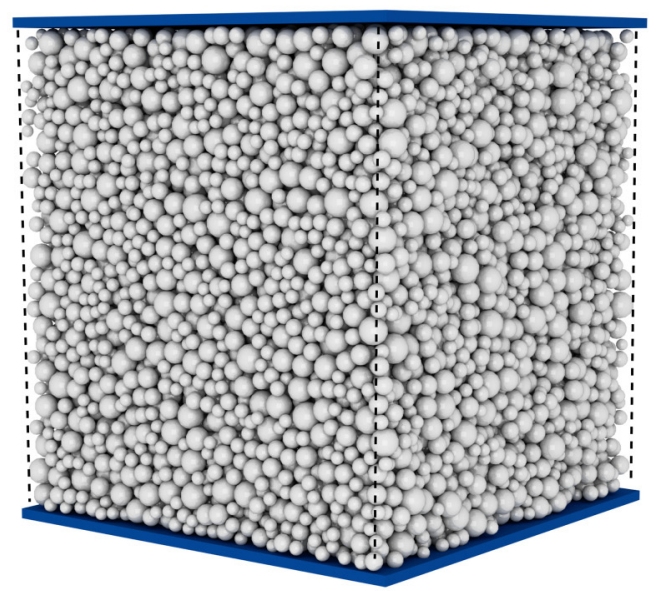

(b)

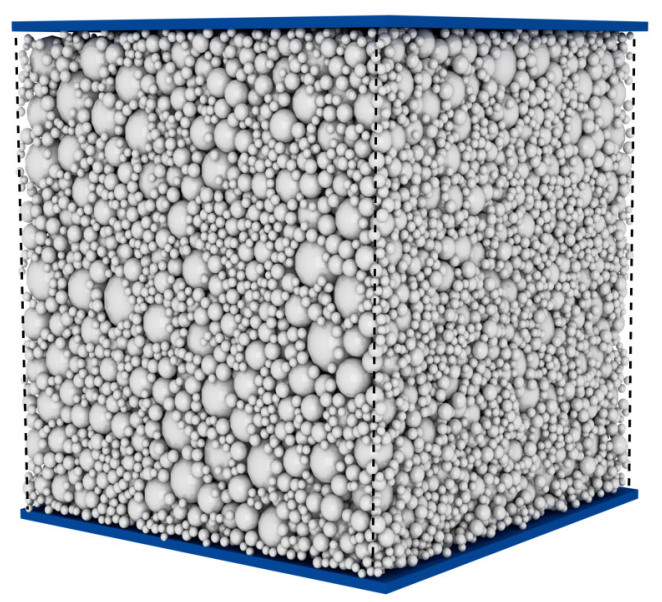

(c)

FIG. 1. Snapshots of samples composed of 20000 spheres with grain size spans corresponding to (a) $S=0.0$, (b) $S=0.4$, and (c) $S=0.8$.

compression process, the gravity as well as the coefficient of friction between spheres and walls were set to 0 . This phase was stopped once a persistent contact network was observed and fluctuations of the number of contacts per particle remained below $0.1 \%$ with respect to the mean value. 


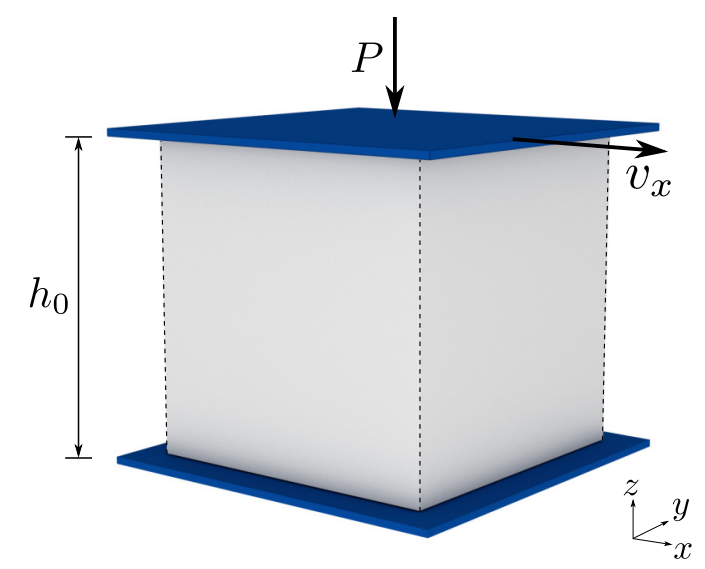

FIG. 2. Schematic representation of the simple shear tests.

Finally, the lateral walls were removed and periodic conditions were set up along the $x$ and $y$ axes. Simple shear tests were undertaken by applying a vertical constant pressure $P$ along the $z$ axis, and a horizontal displacement of the upper wall at constant velocity $v_{x}$ (see Fig. 2). During the shearing tests, friction between particles was set at 0.4 , gravity was maintained to 0 , and particles in contact with the walls were "glued" to them in order to avoid strain localization at the boundaries.

The set of boundary conditions was fixed in order to impose a quasistatic shear regime. The quasistaticity of these tests was measured by means of the inertial number $I=$ $\dot{\gamma} \sqrt{m / P d^{D-2}}$ [43], where $\dot{\gamma}=v_{x} / h_{0}$ is the shear deformation rate with $h_{0}$ the initial height of the sample, $m$ is the mass of a grain with mean diameter $d$, and $D$ is the dimension. Note that the mean diameter that was used for the computation of $I$ corresponds to the one computed from average particle mass, that in the case of a uniform distribution of particle sizes by volume fractions and constant density, corresponds to $\left(d_{\max }+d_{\min }\right) / 2$. Typically, the shear is considered quasistatic if $I<1 \times 10^{-3}$. In our simulations the inertial number was set to $I=1 \times 10^{-4}$. The cumulative shear strain in our tests was measured as $\gamma=x_{w} / h_{0}$, where $x_{w}$ corresponds to the cumulative horizontal displacement of the upper wall. The shearing tests were conducted up to a shear strain of $\gamma=70 \%$ for the samples with polydispersity up to $S=0.6$. For the samples $S=0.7$ and $S=0.8$ (the more demanding in terms of computing resources) the shear strain was preformed up to $\gamma=50 \%$. This level of deformation, as will be shown below, resulted large enough for reaching the steady flow regime in such samples.

In polydisperse granular samples, particle segregation should be avoided or controlled, otherwise local organization may produce crystallization or clustering, and the strain field may present unwanted localizations. Although our layer-bylayer deposit of particles normally prevents segregation at the sample construction stage, we also checked that segregation was not produced during the compaction and shear tests. This was performed by means of Lacey's index defined as $M^{*}=\left(S_{r}^{2}-S^{2}\right) /\left(S_{r}^{2}-S_{0}^{2}\right)$ with $S_{r}$ and $S_{0}$ the statistical variance of the center of the particles for a full random and a completely segregated configuration of grains, respectively

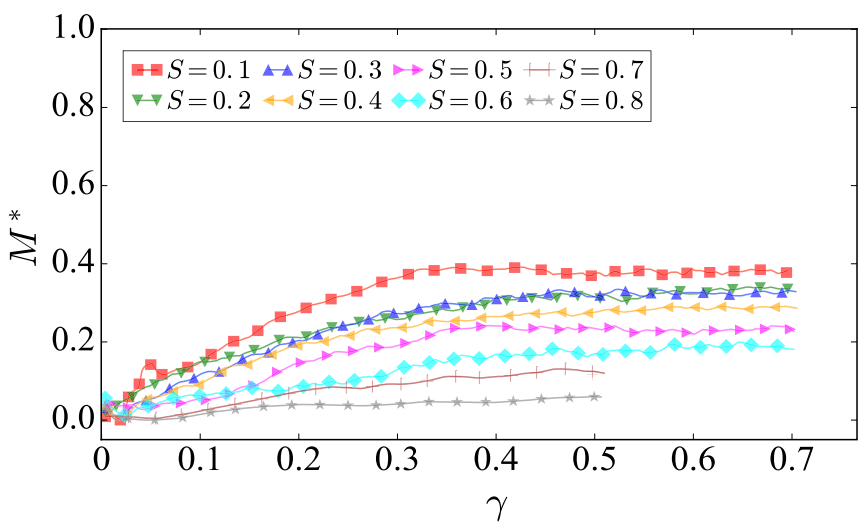

FIG. 3. Evolution of Lacey's parameter for characterizing the relative segregation as a function of the imposed shear strain $\gamma$.

[44]. The Lacey index is a relative parameter that varies between 0 and 1 , being $M^{*}=0$ the case for a completely random distribution of particles, and $M^{*}=1$ the case for a totally segregated sample. The corresponding values of $S_{0}$ were found for each grain size span after deposing the spheres in an ordered sequence by particle size in a box and applying an isotropic compression. The values of $S_{r}$ were computed after ten realizations in which grains were randomly deposited and compressed isotropically. Figure 3 shows the evolution of the Lacey parameter during our shear tests as a function of the shear strain. As can be observed, the random initial arrangement of particles is slightly modified during shearing. We can remark that the particle mixing that is produced during simple shearing and zero gravity conditions can actually prevent very ordered configurations such as the ones created for the computation of the variance $S_{0}$. In effect, the Lacey parameter presents lower values for increasing particle span $S$.

\section{MECHANICAL BEHAVIOR AT THE MACROSCALE}

At the sample scale, we analyze stresses and strains in the steady state in terms of shear strength and solid fraction.

The solid fraction $v$ is defined as the ratio between the volume of particles $V_{s}$ and the volume of the containing box $V$ $\left(v=V_{s} / V\right)$. The shear strength was computed from the stress tensor $\sigma$ of the granular assembly, defined as follows:

$$
\sigma_{i j}=\frac{1}{V} \sum_{c \in V} f_{i}^{c} \ell_{j}^{c},
$$

where $f$ is the contact force and $\ell$ is the branch vector (i.e., the vector joining the centers of particles interacting at contacts $c$ ). Due to the geometry of the simple shear test, the stress state is considered invariant along the $y$ direction. Thus, we may only consider the stresses of the tensor $\sigma$ on the shear plane $x z$. According to the Mohr-Coulomb model, the effective macroscopic friction coefficient $\varphi^{*}$ during shear is given by $\sin \varphi^{*}=q / p$, where $p=\left(\sigma_{1}+\sigma_{2}\right) / 2$ is the mean stress, $q=\left(\sigma_{1}-\sigma_{2}\right) / 2$ is the deviatoric stress, and $\sigma_{1}$ and $\sigma_{2}$ are the principal values of the stress tensor on the shear plane $[45,46]$.

Figures 4(a) and 4(b) present the evolution of $v$ and $q / p$, respectively, for the nine samples as a function of the shear 
(a)

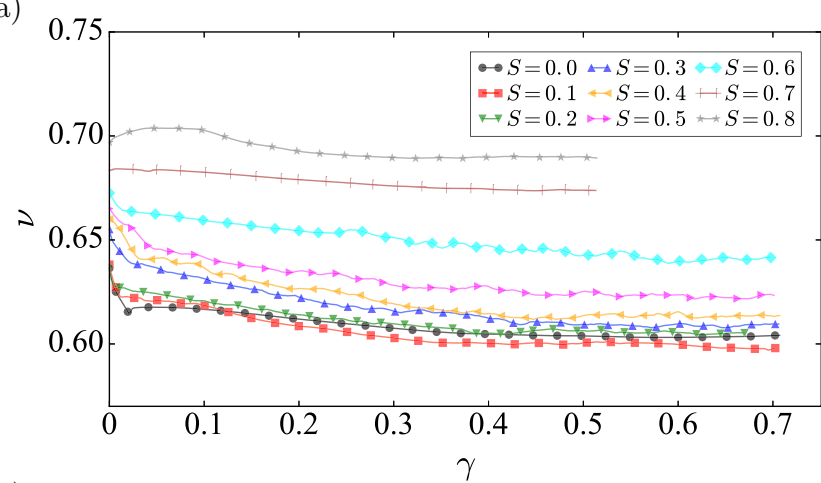

(b)

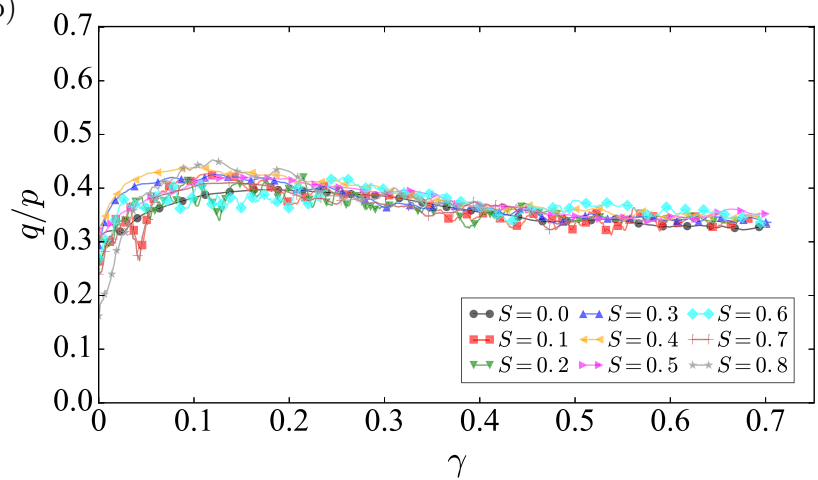

FIG. 4. Evolution of the packing fraction $v$ (a) and the shear strength $q / p$ (b) for each value of $S$, as a function of the shear strain $\gamma$.

strain $\gamma$. We can observe that solid fraction evolves rapidly at the beginning of the tests and smoothly tends to a steady value. As the particle size span increases, small grains can fill the pores around bigger particles increasing the average density. At the same time, we remark that the shear strength $q / p$ increases with $\gamma$ before finding a steady value at values of $\gamma$ greater to $35 \%$ for all grain size dispersions. In effect, we consider that a steady shear state is reached for $\gamma>0.4$ as only small fluctuations of strength and solid fraction are observable around their mean values. In order to characterize this steady state, the sample properties that are presented in the following are computed for the last $30 \%$ of cumulative shear strain.

Figures 5(a) and 5(b) show the average solid fraction $v^{*}$ and the macroscopic friction angle $\sin \varphi^{*}$ defined from the mean value of $q / p$ in the steady state, both as a function of the size span $S$. We observe that the solid fraction increases nonlinearly with the size span. In contrast, shear strength is almost independent of $S$, evidencing that packing density does not affect the mechanical strength of these granular assemblies. Although these observations suggest that the macroscopic behavior of granular materials are unrelated to space dimensionality, we will show that (1) the microstructure and the force transmission can only be fully appreciated by means of 3D simulations, and (2) the mechanisms at the origin of the shear strength are fundamentally different in each of our samples and differ from their analog cases when using disks in 2D simulations.

Another interesting part of the stress analysis in the case of periodic simple shear tests concerns the variation of the

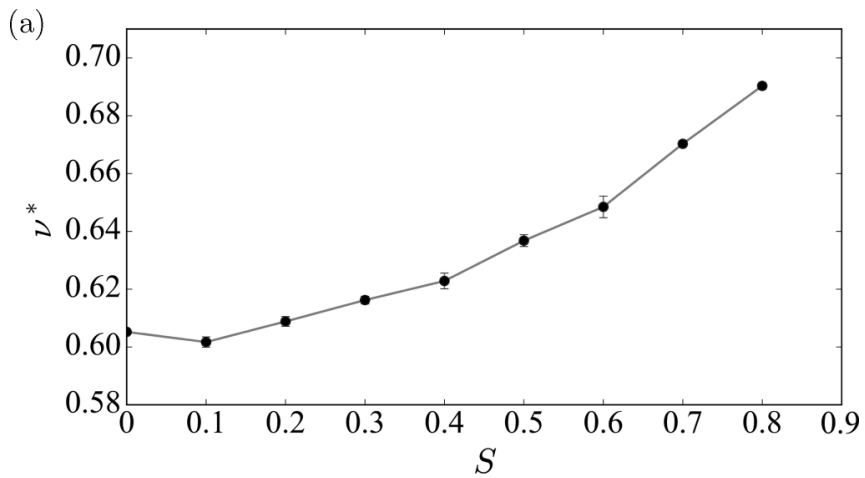

(b)

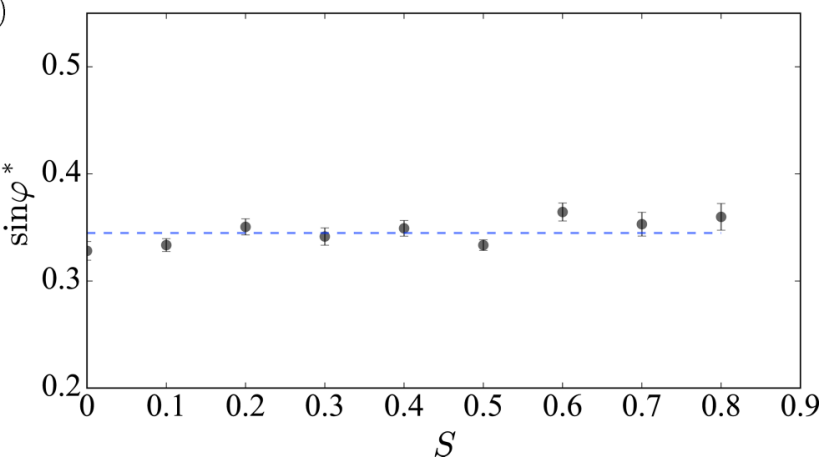

FIG. 5. Mean solid fraction $v^{*}$ (a) and macroscopic friction $\sin \varphi^{*}$ (b), averaged in the steady state as a function of $S$. Error bars present the standard deviation of the data. The dashed line shows the mean macroscopic friction along all packings.

stress tensor components. This analysis, seldom seen in the study of granular flows, shows in our case that $\sigma_{x x}$ actually differs from $\sigma_{y y}$ as the shear deformation is imposed. Note that $\sigma_{x x}=\sigma_{y y}=\sigma_{z z}$ at the beginning of the shearing given the isotropic compression stage. However, we observe that the ratio $\sigma_{x x} / \sigma_{y y}$ drifts from 1 and reaches a steady value independent of the polydispersity around 1.1. On the other hand, $\sigma_{x x} / \sigma_{z z}$ differs from 1 during the nonsteady and transient shear phase but it is rapidly stabilized around 1 once the steady state is reached (note that $\sigma_{z z}$ equals the imposed vertical pressure $P$ ). Our data are consistent with previous work where a normal stress difference in shear flow is observed in general, although sometimes reported to be negligible [46].

\section{GRANULAR MICROSTRUCTURE}

In this section, we consider various statistical descriptors allowing us to quantify the effect of grain size span on the geometrical organization (i.e., fabric) and the force transmission within the packings.

\section{A. Particle connectivity}

Figure 6 shows snapshots of floating particles [(a), (b)], i.e., particles with none or only one contact, and a gray level map revealing the particle connectivity [(c), (d)] for a monodisperse and a polydisperse sample in the steady state. In the case of $S=0$, floating particles are rare and mostly isolated. As $S$ increases, we easily observe that floating particles 


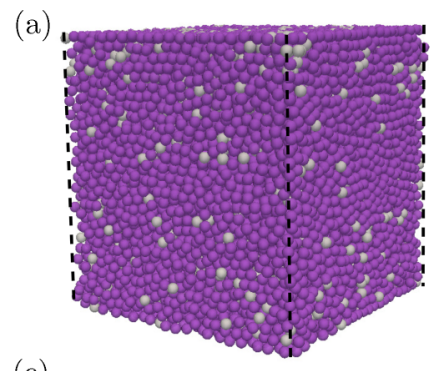

(c)

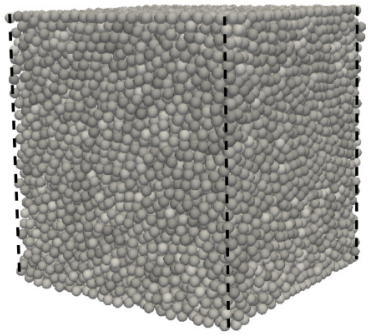

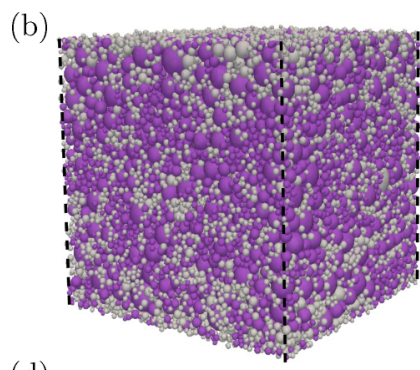

(d)

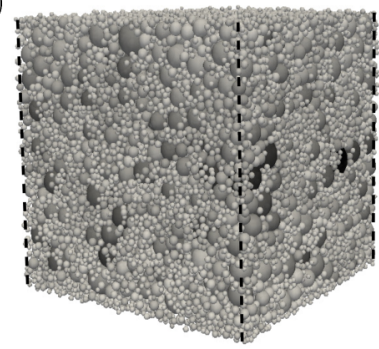

FIG. 6. The floating particles (i.e., particles with one or no contacts) in gray [(a), (b)] and color (gray) map of particle connectivity [(c), (d)], for $S=0.0$ [(a), (c)], and $S=0.8$ [(b), (d)], in the steady state. The gray scale is proportional to coordination number.

correspond to the smallest spheres while the large particles have an increasing number of contacts.

At the first order, the connectivity of the contact network is characterized by the proportion $c_{0}$ of floating particles and the average number of contacts, also known as coordination number, defined as $Z=2 N_{c} /\left\{n\left(1-c_{0}\right)\right\}$ with $N_{c}$ the number of active contacts (i.e., those participating in the transmission of forces, in which the normal contact force is greater than 0 ), and $n$ the number of particles. The factor $\left(1-c_{0}\right)$ excludes the floating particles of this computation. These two parameters are shown in Fig. 7(a) as a function of $S$. We see that the proportion of floating particles increases nonlinearly from $c_{0} \simeq 0.05$ for $S=0.0$ to $c_{0} \simeq 0.55$ for $S=0.8$. In addition, the inset of Fig. 7(a) shows that the coordination number slightly declines with $S$ from $Z \simeq 4.5$ to around 3.9. In other words, for larger size spans, around half of the particles do not take part in the contact network. Those particles participating in the network are conversely less connected than grains in the monodisperse packing. This observation is quite surprising considering that packings are more compact as $S$ increases. Nevertheless, we have to recall that the particle size distribution is uniform by volume fractions. This means that, as $S$ is increased, the proportion of small particles increases faster than those of bigger size and $Z$ can be strongly influenced by the connectivity of the small particle classes. The reduction in the average coordination number for large $S$ may be explained as well by an increment of semistable particles having only two contacts as shown by Agnolin and Roux [47].

Let us consider the proportion of floating particles per particle class $C_{0_{d r}}$ as a function of the reduced diameter $d_{r}$ defined as

$$
d_{r}=\frac{d-d_{\min }}{d_{\max }-d_{\min }} .
$$

The reduced diameter may vary between 0 and 1 , and represents the relative size scale of a grain of diameter $d$, with

(a)

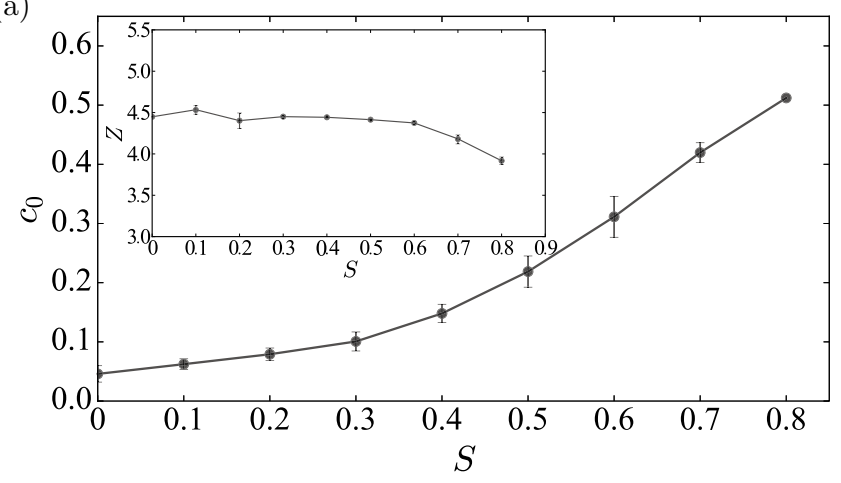

(b)

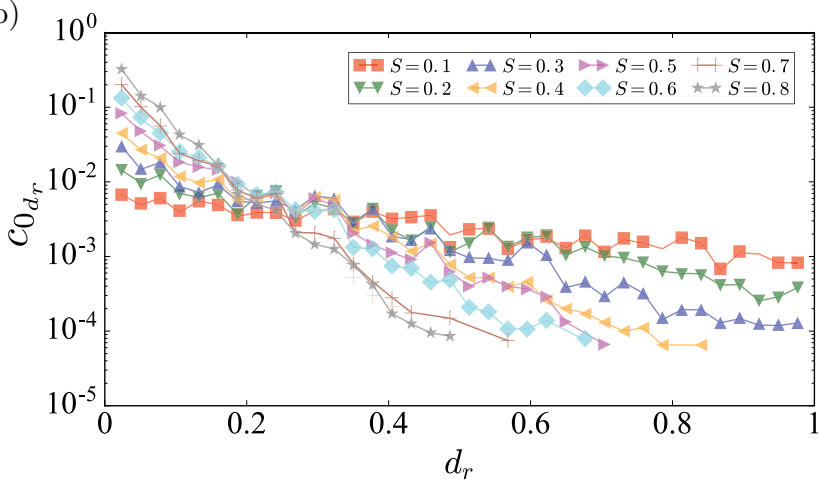

FIG. 7. (a) Proportion $c_{0}$ of floating particles and coordination number $Z$ (inset) as a function of size span. Error bars display the standard deviation of the data. (b) Proportion of floating particles as a function of the reduced particle diameter $d_{r}$.

respect to the maximum and minimum sizes of particles in the sample. Figure 7(b) presents the proportion $C_{0_{d r}}$ as a function of the reduced diameter for the different values of $S$. As expected, the smallest granulometric classes are those that contribute the most to the rattler population. In contrast, for particle size spans $S>0.4$ the largest particle class is always being part of the granular backbone.

In Fig. 8(a) we consider the evolution of the coordination number $Z_{d_{r}}$ as a function of the reduced diameter and the different size spans. Interestingly, we observe a linear dependence between $Z_{d_{r}}$ and $d_{r}$, with an increasing slope as $S$ increases. This confirms again that smaller particles have fewer contacts than particles of larger sizes. In contrast, the class of larger particles is more connected with coordination values $Z_{d_{r}}$ up to 16 for the largest size span tested.

In order to have a complete outlook of the evolution of the number of contacts per particle, we show in Fig. 8(b) the average proportion $P_{k}$ of having $k$ contacts per sphere as a function of the particle size span. It is worth mentioning that for $k \leqslant 2$, the proportion $P_{k}$ increases with $S$ while for $4 \leqslant$ $k \leqslant 7, P_{k}$ decreases with size span. Interestingly, $P_{3}$ seems to reach global maximum for $S=0.5$. Finally, for $k \geqslant 8, P_{k}$ slightly increases as richer contact configurations are allowed once the smallest grains can enter the pores left by the largest particles.

The description of the microstructure in terms of the coordination number and connectivity by particle class provide a first picture on the effect of grain size span. It is remarkable that the trends observed here in 3D simulations hold up the 
(a)

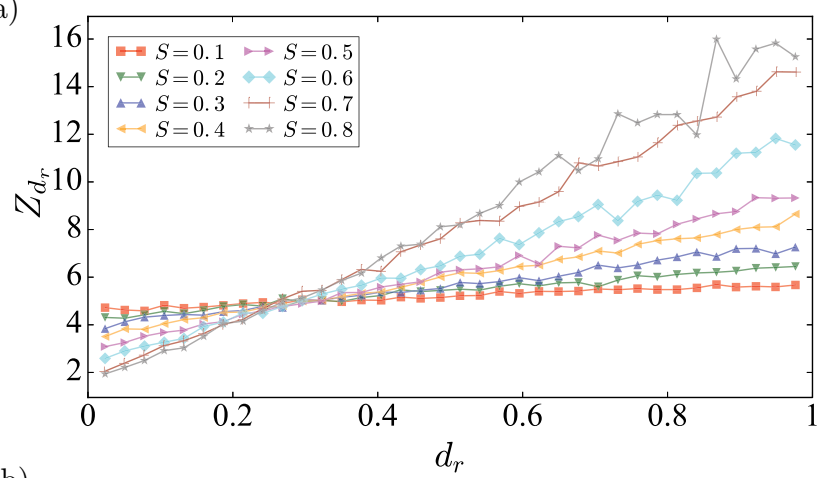

(b)

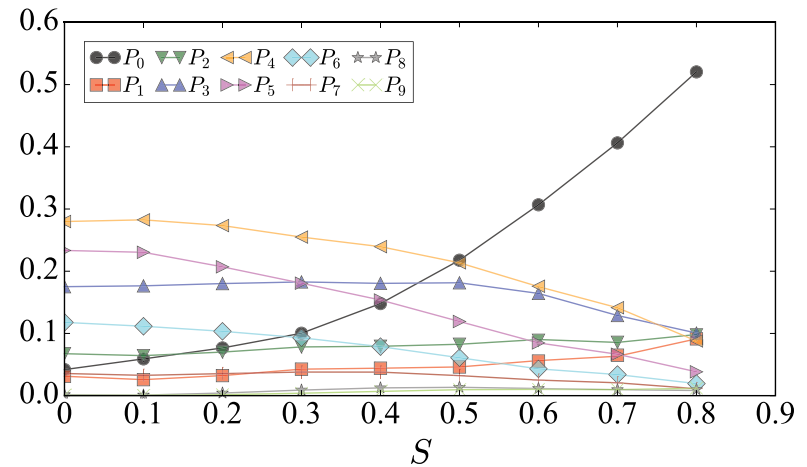

FIG. 8. (a) Average number of contacts $Z_{d_{r}}$ as a function of reduced diameter $d_{r}$ for all values of size span $S$. (b) Proportion $P_{k}$ of particles with $k$ contacts as a function of the grain size span $S$.

results observed by Nguyen et al. by means of 2D simulations [9]. In order to complete this picture of particle size span effects, we analyze the interparticle forces in the following.

\section{B. Force transmission and friction mobilization}

At the contact scale, the local geometry and the mechanical equilibrium associated with two touching particles can be characterized by means of the branch vector $\boldsymbol{\ell}$ (i.e., the vector joining the centers of the particles), and the contact force $f$ (see Fig. 9). At this scale, a local framework $(\boldsymbol{n}, \boldsymbol{t})$ is defined, being $\boldsymbol{n}$ the unit vector perpendicular to the contact plane and $\boldsymbol{t}$ an orthonormal unit vector oriented along the tangential force. Then, we have $\ell=\ell \boldsymbol{n}$, where $\ell$ is the length of the branch

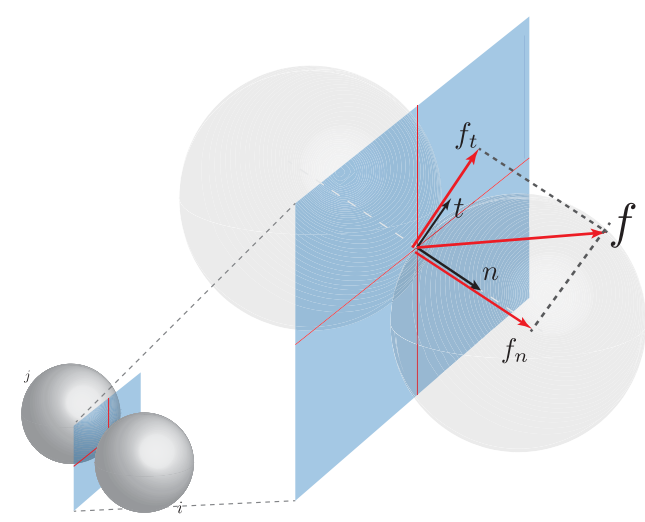

FIG. 9. Local geometry. (a)

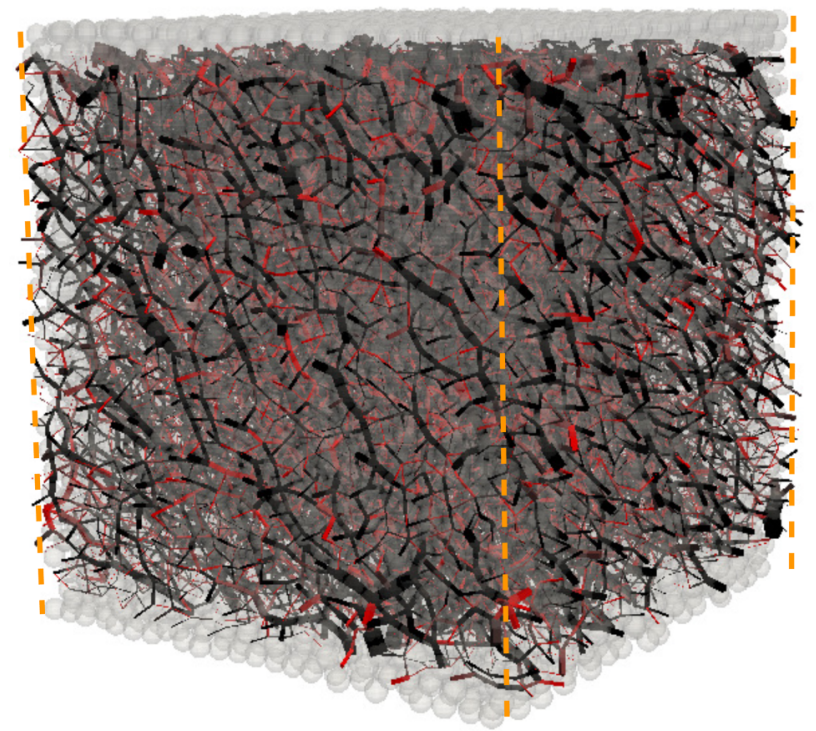

(b)

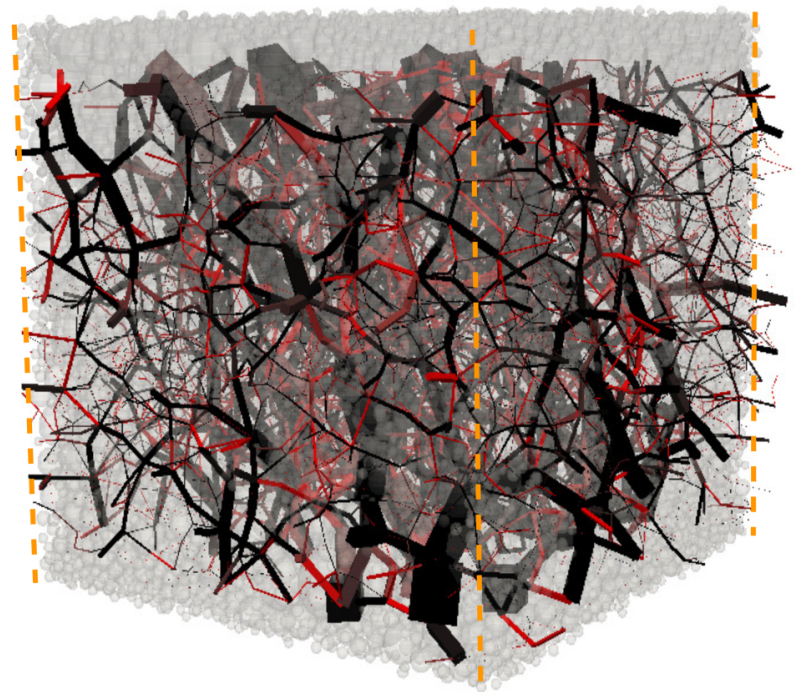

FIG. 10. Snapshot of normal forces in the samples for (a) $S=$ 0.0 and (b) $S=0.8$. Line thickness is proportional to the normal force intensity. Mobilized forces (where $\left|f_{t}^{c}\right|=\mu f_{n}^{c}$ at the corresponding contact $c$ ) are displayed in red.

vector, and $\boldsymbol{f}=f_{n} \boldsymbol{n}+f_{t} \boldsymbol{t}$, where $f_{n}$ and $f_{t}$ are the normal and tangential contact forces.

Given the above definitions, Fig. 10 shows two maps of normal forces for $S=0.0$ (a) and $S=0.08$ (b), respectively. Forces are represented with bars along the branch vectors, their thickness is proportional to the intensity of the normal contact force, and mobilized contacts (i.e., contact where $\left.\left|f_{t}\right|=\mu f_{n}\right)$ are displayed in red. Visual inspection reveals the anisotropic and inhomogeneous nature of the contact and force networks. In particular, these features seem to be amplified at larger values of $S$ where we can find stronger forces and many more small forces.

The inhomogeneity of contact forces in granular media has been intensively investigated in experiments and in numerical simulations using monodisperse or slightly disperse spherical and nonspherical particles [48-59]. However, it is still seldom seen for polydisperse packings. As typically observed, 
(a)

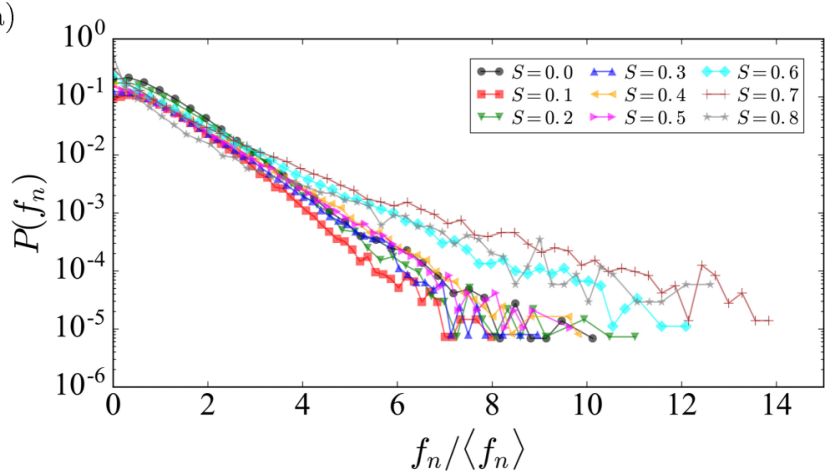

(b)

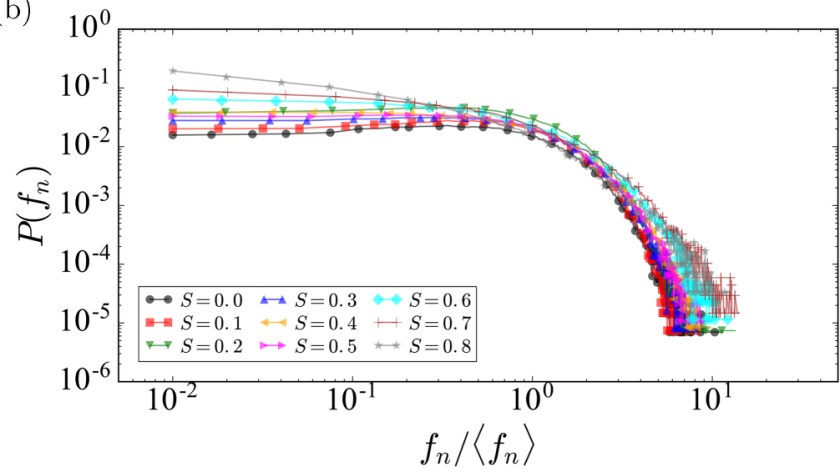

FIG. 11. Probability density functions of normal forces in loglinear (a) and log-log scales (b).

the probability density function (PDF) of normal contact forces presents two characteristics: (1) the PDF is roughly a decreasing exponential function for forces above the mean and (2) in the range forces below the mean, the probability of having very small forces does not decline to zero.

In Fig. 11 we show the PDF of normal forces in our tests after a normalization by the mean normal force $\left\langle f_{n}\right\rangle$. We present these distributions in a log-linear (a) and a log-log scale (b), for all the grain size spans. We remark that the PDFs are significantly broader as $S$ increases. For instance, the maximum normal force varies from 5 times the mean force for $S=0.0$ to nearly 14 times the mean force for $S=0.8$. We also observe an increasing number of contacts carrying weak forces (below the mean) as $S$ increases. These distributions clearly indicate that the inhomogeneity in force transmissions are more important for samples composed of particles of different sizes. In particular, at large particle size spans, this phenomenon is heightened as fewer particles are participating in the contact network.

Force transmission is naturally related to the geometrical properties of the contact such as the branch vector. As the size span $S$ increases, the range of branch lengths $\ell$ becomes naturally broader and it is easy to show that the ratio $\ell / R_{\max }$, with $R_{\max }$ the maximum particle radius, varies from $2(1-$ $S) /(1+S)$ to 2 . The lower limit corresponds to contacts between the smallest spheres while the upper limit represents contacts between two of the biggest spheres. For instance, $\ell / R_{\max }=2$ for $S=0.0$, whereas $\ell / R_{\max } \in[\simeq 0.22 ; 2]$ for $S=0.8$. Then, we may wonder whether a correlation exists between the branch length and the corresponding average forces at the contacts. (a)

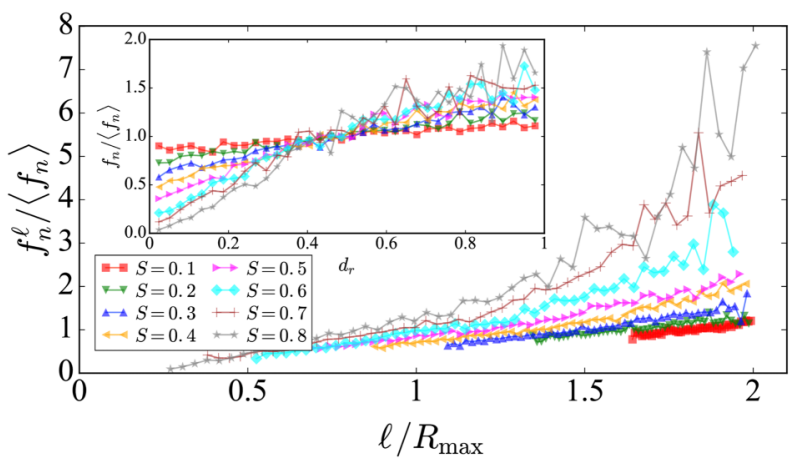

(b)

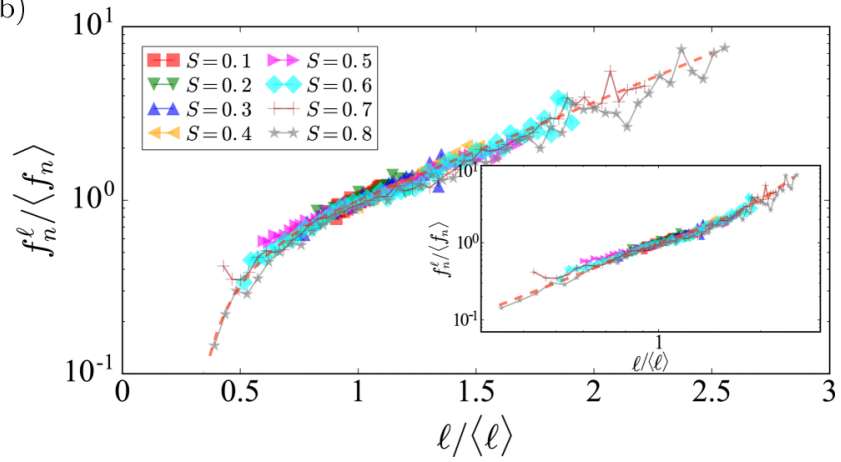

FIG. 12. (a) Normal forces normalized by the mean $\left\langle f_{n}\right\rangle$ as a function of the branch length normalized by the maximum radius $R_{\max }$ and (in the inset) as a function of the reduced diameter $d_{r}$. (b) Normal forces normalized by the mean $\left\langle f_{n}\right\rangle$ as a function of the branch length normalized by the mean branch $\langle\ell\rangle$. A log-log representation is shown in the inset. The (brown) dashed lines present the best fits by means of a power and exponential relation for $\ell /\langle\ell\rangle<$ 1 and $\ell /\langle\ell\rangle \geqslant 1$, respectively.

Figure 12(a) shows the average normal force $f_{n}^{\ell}$, calculated by taking the average force in a class of contacts in small intervals $[\ell-\Delta \ell / 2, \ell+\Delta \ell / 2]$ as a function of $\ell / R_{\max }$ for all values of $S$. A general observation is that $f_{n}^{\ell}$ increases with $\ell$. For $S \leqslant 0.5, f_{n}^{\ell}$ barely varies around the mean $\left\langle f_{n}\right\rangle$, whereas at larger values of $S$ we observe two well-defined zones: (1) the class of the shortest branch vector $\left(\ell<R_{\max }\right)$, which concentrates, on average, the forces below the mean $\left\langle f_{n}\right\rangle$, and (2) the class of the longest branch vectors $\left(\ell>R_{\max }\right)$, which carry stronger forces.

Considering that particle size is naturally encoded in the branch length, we can expect that larger particles are capturing the stronger force chains while small particles concentrate small forces. This is well verified in the inset of Fig. 12(a), in which we present the average normal force $f_{n}^{d_{r}}$, calculated by taking the average force in a class of particle in small intervals $\left[d_{r}-\Delta d_{r} / 2, d_{r}+\Delta d_{r} / 2\right]$ as a function of $d_{r}$ for all values of $S$. Such averages show that, as the size span increases, smaller particles support smaller forces whereas bigger particles support stronger forces. In particular, this representation highlights that, regardless of $S$, particles of mean size $d_{r} \sim$ 0.5 are persistently supporting the mean force $\left\langle f_{n}\right\rangle$. Thus, we can expect a similar behavior as a function of the average branch $\ell$. Figure 12(b) shows the average normal force $f_{n}^{\ell}$ as a function of the branch length normalized by the mean branch $\langle\ell\rangle$ in lin-log scale and in log-log scale (inset). We see that 
(a)

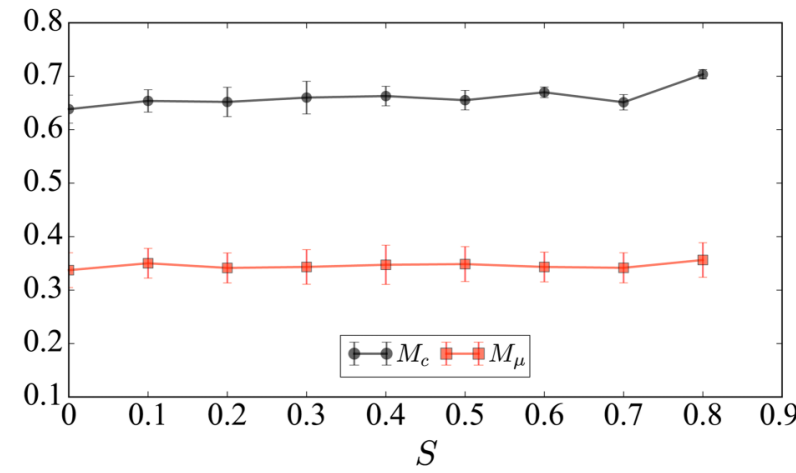

(b)

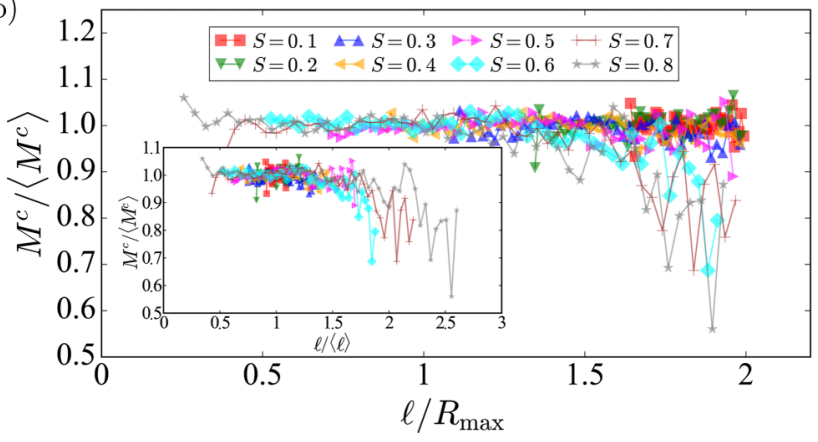

FIG. 13. (a) Friction mobilization, averaged in the steady state, as a function of size span $S$. (b) Friction mobilization index $M_{\mu}$ normalized by the mean $\left\langle M_{\mu}\right\rangle$ as a function of the normalized branch length $\ell / R_{\max }$ (the inset shows the same plot as a function of the branch length normalized by the mean branch $\langle\ell\rangle$.

all data collapse on a master curve (dashed line) following an exponential law $f_{n} /\left\langle f_{n}\right\rangle \propto e^{\ell /\langle\ell\rangle}$ for $\ell>\langle\ell\rangle$ and a power law $f_{n} /\left\langle f_{n}\right\rangle \propto\left\{\left(\ell-\ell_{\min }\right) /\langle\ell\rangle\right\}^{\alpha}$ with $\ell_{\min }=2 d_{\text {min }}$ (i.e., two times the diameter of the smallest particle in the sample), for branches below the mean. These relations are quite interesting because they allow one to predict the potential contact forces that a couple of particles are transmitting in any polydisperse sample just by knowing the average contact force whose value may be estimated once the pressure $P$ is set and the size of the sample reaches the steady state.

Parallel to the transmission of normal contact forces, it is important to analyze the distribution of tangential contact forces within the system. On average, the friction forces as well as the number of sliding contacts, also called "mobilized contacts," increase with shear stress. The mobilization of a contact occurs for the subset where $\left|f_{t}\right|=\mu f_{n}$ (see also Fig. 10). The degree of mobilization of friction forces is a state parameter in granular materials that can be defined either by the average normal and tangential forces as $M_{\mu}=$ $\left\langle\left|f_{t}\right|\right\rangle /\left(\mu\left\langle f_{n}\right\rangle\right)$, or by means of individual contact forces as $M_{c}=\left\langle\left|f_{t}\right| /\left(\mu f_{n}\right)\right\rangle$. Note that, the first definition is closest to the Mohr-Coulomb criterion than the second one. A general observation, performed by means of numerical simulations, is that $M_{c} \simeq 2 M_{\mu}[59,60]$. From our numerical experiments, we can remark that such relation is still valid in $3 \mathrm{D}$.

Figure 13(a) shows $M_{c}$ and $M_{\mu}$ in the steady state, as a function of $S$. We see that the mobilization degrees are independent of $S$ which is consistent with the invariance of shear strength at the macroscopic scale (see Sec. III). The proportion

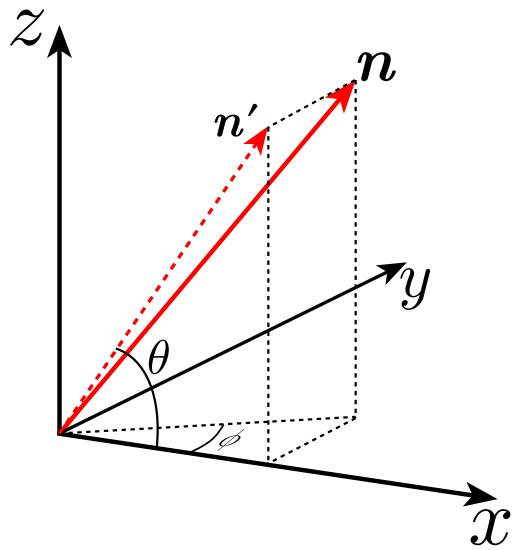

FIG. 14. Normal contact orientation given the azimuthal angle $\phi$ and the angle created by the projection of the contact direction on the shear plane $(\theta)$.

of sliding contacts (not shown here) is also independent of $S$ and remains around $20 \%$ in all simulations. In order to investigate the distribution of mobilized contacts within the sample, we plot in Fig. 13(b) the average friction mobilization index by branch class $M_{\mu}^{\ell}$ normalized by the mean $M_{\mu}$, as a function of $\ell$ normalized by $R_{\max }$ and by $\langle\ell\rangle$ (in inset). This plot shows that, while $S \leqslant 0.5$, the associated mean mobilization for each contact class is very similar to the global mean mobilization $\left\langle M_{\mu}\right\rangle$. In contrast, as $S$ increases, the class of largest branches mobilizes relatively fewer contacts as $M_{\mu}^{\ell}$ is slightly smaller than the mean $\left\langle M_{\mu}\right\rangle$. These observations point out that the mechanical equilibrium of the bigger particles is principally controlled by the compressive contact forces.

In the following section, we introduce higher order parameters in order to enrich the current microscopic characterization. In particular, given that the stress tensor [Eq. (2)] can be rewritten in terms of angular distributions of contact forces and branches [58], we are able to obtain a full description of the previous structural descriptors according to the contacts orientations in space.

\section{Fabric and force anisotropies}

The fabric in granular materials refers to a second-order analysis of the topology of the contact network and force chains where scalar quantities are considered along a direction (e.g., the normal contact vector). Let us consider $\mathcal{S}(\Omega)$ the set of contact vectors pointing in the direction $\Omega=(\theta, \phi)$, with $\theta$ the angle of the normal contact vector projected on the shear plane and $\phi$ the azimuthal angle (see Fig. 14).

The fabric of the contact network can be represented by the probability density function $P_{n}(\Omega)$ defined as $[61,62]$

$$
P_{n}(\Omega)=\frac{N_{c}(\Omega)}{N_{c}},
$$

where $N_{c}$ is the total number of contacts and $N_{c}(\Omega)$ is the number of contacts pointing in the direction $\Omega$. 


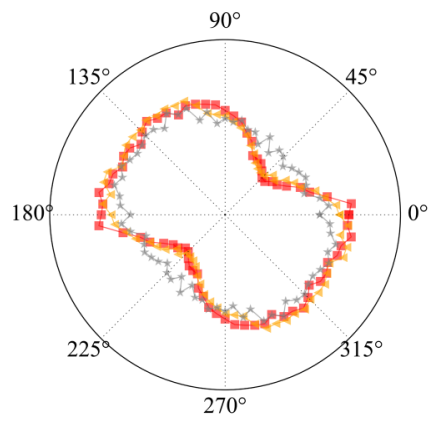

(a)

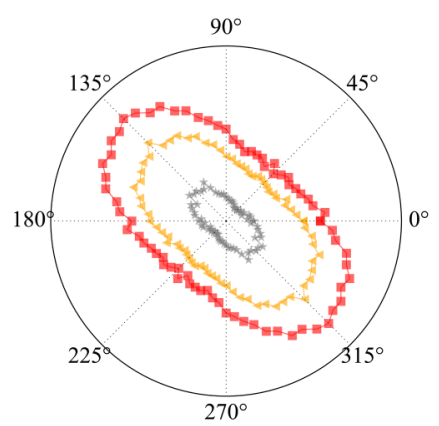

(c)

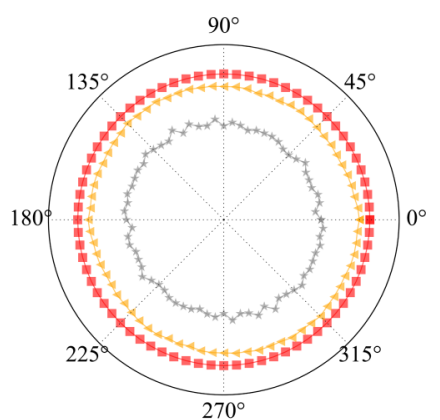

(b)

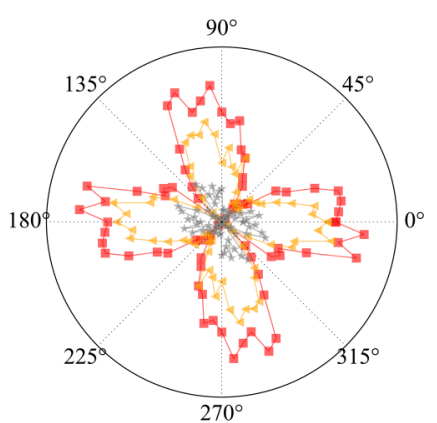

(d)

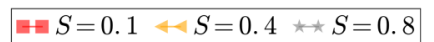

FIG. 15. Polar representation of the functions (a) $P_{n}(\theta)$, (b) $\langle\ell\rangle(\theta)$, (c) $\left\langle f_{n}\right\rangle(\theta)$, and (d) $\left\langle f_{t}\right\rangle(\theta)$ for $S=0$ (red squares), $S=0.4$ (yellow triangles), and $S=0.8$ (gray stars).

Along with $P_{n}(\Omega)$, the branch length, the normal and tangential contact forces can be expressed as a function of the orientation $\Omega$ as $[58,63]$

$$
\begin{aligned}
\langle\ell\rangle(\Omega) & =\frac{1}{N_{c}(\Omega)} \sum_{c \in S(\Omega)} \ell^{c}, \\
\left\langle f_{n}\right\rangle(\Omega) & =\frac{1}{N_{c}(\Omega)} \sum_{c \in S(\Omega)} f_{n}^{c}, \\
\left\langle f_{t}\right\rangle(\Omega) & =\frac{1}{N_{c}(\Omega)} \sum_{c \in S(\Omega)} f_{t}^{c} .
\end{aligned}
$$

As was mentioned before, the symmetry of the shear test along the $y$ axis allows one to neglect the azimuthal angle and consider only the projections of contact orientations on the shear plane. Thus, the normal contact orientation is represented by the vector $n^{\prime}$ (see Fig. 14) with orientation $\theta$ and the contact tangent vector $t^{\prime}$ is orientated at $\theta+\pi / 2$ on the shear plane as well.

Figure 15(a) displays a polar representation of functions $4,5,6$, and 7 in the shear plane as a function of the angle $\theta$ in the steady state for three different values of $S$. For $P_{n}(\theta)$ and $\left\langle f_{n}\right\rangle(\theta)$, we observe an anisotropic behavior where the peak value occurs, on average, along the major principal stress direction $\theta_{\sigma} \simeq 3 \pi / 4$. The peak values for $\left\langle f_{t}\right\rangle(\theta)$ occur at $\theta_{f_{t}} \simeq \pi / 2$. For $\langle\ell\rangle(\theta)$ the probability density functions are nearly isotropic.

These four functions are $\pi$ periodic and, as frequently shown in literature, they can be well approximated by

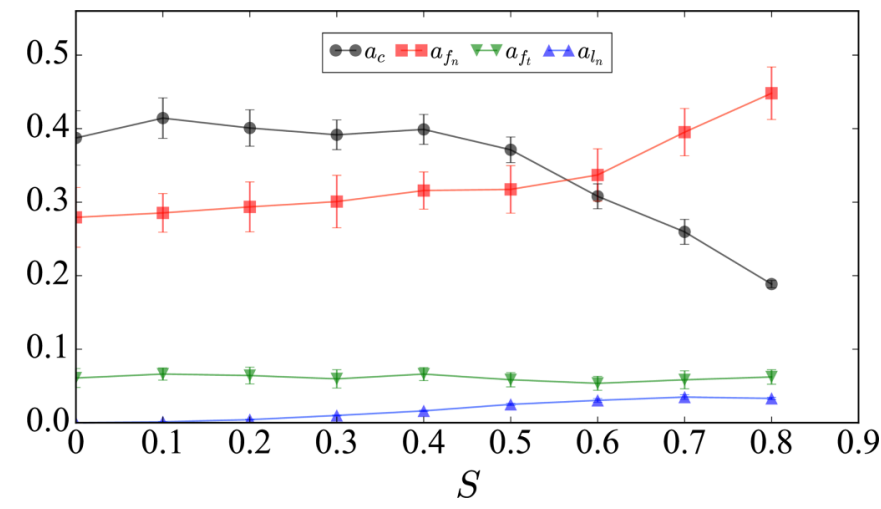

FIG. 16. Evolution of the contact $\left(a_{c}\right)$, the branch $\left(a_{\ell}\right)$, and the forces $\left(a_{f_{n}}, a_{f_{t}}\right)$ anisotropies as a function of the particle size span $S$. Error bars show the standard deviation of the data.

second-order Fourier expansions as follows:

$$
\begin{aligned}
P_{n}(\theta) & =\frac{1}{\pi}\left\{1+a_{c} \cos 2\left(\theta-\theta_{c}\right)\right\}, \\
\langle\ell\rangle(\theta) & =\langle\ell\rangle\left\{1+a_{l} \cos 2\left(\theta-\theta_{\ell}\right)\right\}, \\
\left\langle f_{n}\right\rangle(\theta) & =\left\langle f_{n}\right\rangle\left\{1+a_{f_{n}} \cos 2\left(\theta-\theta_{f_{n}}\right)\right\}, \\
\left\langle f_{t}\right\rangle(\theta) & =\left\langle f_{n}\right\rangle\left\{a_{f_{t}} \sin 2\left(\theta-\theta_{f_{t}}\right)\right\},
\end{aligned}
$$

where $a_{c}, a_{\ell}, a_{f_{n}}$, and $a_{f_{t}}$ are the contact, branch-length, normal, and friction force anisotropies. Additionally, $\theta_{c}, \theta_{\ell}, \theta_{f_{n}}$, and $\theta_{f_{t}}$ are the main orientations for each angular distribution. Note that, even if these anisotropies and main orientations can be extracted from the angular distributions, it is more convenient to use the fabric and force tensors defined, for example, in [58].

Figure 16 displays the averaged anisotropies in the steady state, as a function of $S$. We see that $a_{c}$ declines from 0.4 to 0.2 as $S$ increases. The decrease of $a_{c}$ in the steady state is compatible with the fact that large particles are being surrounded by an increasing number of small particles (see Sec. IV A). The increasing connectivity of the biggest particles imply a higher dispersion of contact orientations. In the case of the normal force anisotropy, $a_{f_{n}}$ increases with $S$ from 0.3 to 0.45 while the branch-length anisotropy $a_{\ell}$ slightly increases from 0 to 0.05 . The large variation of $a_{f_{n}}$ with $S$ is consistent with the fact that the probability density function of normal forces gets broader with $S$. The increase of $a_{\ell}$ is related with the preference of the biggest particles to get aligned along strong force chains. On the other hand, we observe that the tangential force anisotropy is constant with $S$. Indeed, by integrating Eq. (8d) in the range $[0, \pi / 2]$, we show that the friction force anisotropy is related to the friction mobilization as $\left\langle M_{\mu}\right\rangle=2 a_{f_{t}} / \mu[37,58]$.

The force and fabric anisotropies are, in fact, at the origin of shear strength in granular materials. It has been shown that the expression of the stress tensor [Eq. (2)] together with the Fourier expansions [Eq. (8)] lead to the following relation in the steady state $[37,58,63]$ :

$$
\sin \varphi^{*} \simeq \frac{1}{2}\left(a_{c}+a_{\ell}+a_{f_{n}}+a_{f_{t}}\right) .
$$




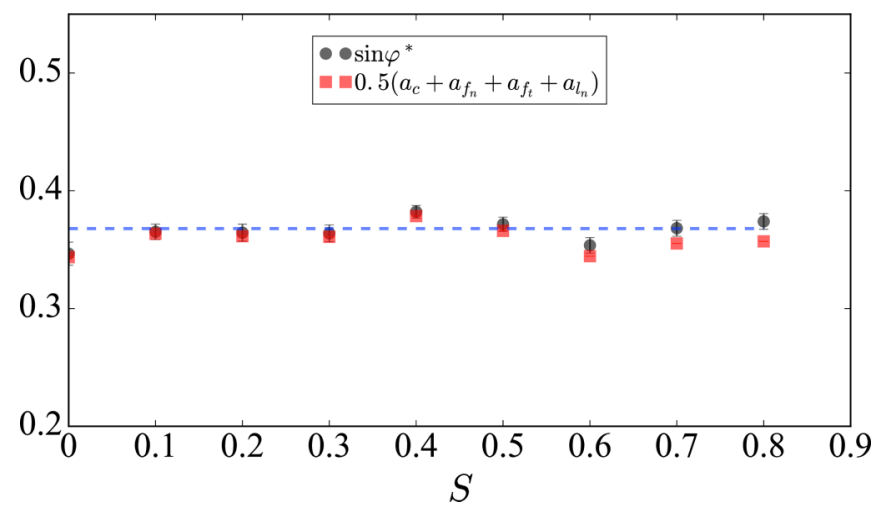

FIG. 17. Normalized shear stress $\sin \varphi^{*}$ in the residual state as a function of $S$ (black points) with the harmonic approximation (red squares) given by Eq. (9). Error bars represent the standard deviation of the data.

The predicted values of $\sin \varphi^{*}$ by this equation, as well as the values measured at the macroscopic scale are shown in Fig. 17 as a function of $S$.

Besides predicting very well the friction angle for all the values of $S$, Eq. (9) allows one to predict very well the friction angle for all values of $S$. On the other hand, it reveals the origins of shear stress in a granular system: (1) the fabric anisotropies $a_{c}$ and $a_{\ell}$, related to the contact and branch vector, (2) the normal force anisotropy $a_{f_{n}}$, related to force transmission, and (3) the tangential force anisotropy $a_{f_{t}}$, related to friction mobilization. We can now determine that the independence of $\sin \varphi^{*}$ with respect to particle size span emerges from the compensation between the drop of contact anisotropy $\left(a_{c}\right)$, the increment in force anisotropy $\left(a_{f_{n}}\right)$, and a slight gain in branch anisotropy $\left(a_{\ell}\right)$.

To conclude, it is worth mentioning that the results presented here are consistent with those obtained by Voivret $e t$ al. [31] and by Nguyen et al. [9] by means of 2D simulations using disks and polygons, respectively. Nevertheless, they observed that branch-length anisotropy has a larger influence in shear strength than force anisotropy, especially at large values of $S$. Instead, we show in this paper that the variation of such anisotropy is rather small in 3D simulations. A detailed analysis of anisotropies by granulometric class also revealed that such evolution of $a_{f_{n}}$ and $a_{\ell}$ does not belong to a preferential particle size. Instead, the assembly of particles participating in the stress transmission present similar microstructural processes, impeding to completely discard particles below or above a certain granulometric class from the shear strength analysis.

\section{SUMMARY AND PERSPECTIVES}

In this paper, we presented a systematic analysis of the effect of size span on the rheological properties of sheared granular materials composed of spherical particles using 3D contact dynamic simulations. Considering a uniform size distribution by volume fractions, the size span $S$ was systematically varied from 0 (monodisperse packing) to $S=0.8$ (polydisperse packing). The macroscopic and microstructural properties of several packings under simple shear conditions were analyzed as a function of $S$.

It was shown that the shear strength in the steady flow state is independent of $S$, whereas the packing fraction increases nonlinearly with the grain size span. This finding confirms previous results using disks and polygons in $2 \mathrm{D}$ simulations $[9,31]$. The microstructure was analyzed in terms of particle connectivity, force transmission, and anisotropies of the fabric and the force networks.

An increasing span $S$ produces a decrease of the number of particles participating in the contact network while the biggest particles are surrounded by several small ones. At the same time, we observed a clear correlation between contact forces and branch vectors evidencing that large particles are strongly involved in force transmission and the mechanical equilibrium. In consequence, while the fabric anisotropy declines with $S$, such drop is perfectly compensated by an increment of normal force anisotropy and branch anisotropy. This tradeoff between geometrical and force anisotropies explains the independence of the shear strength with respect to the grain size span $S$ at the macroscopic scale.

Our findings confirm that, at the first order, an "average particle size" is sufficient for modeling the macroscopic behavior of granular media. Even so, we have to keep in mind that the microscopic mechanisms underlying the shear strength are deeply particle size dependent. This fact may have an important impact in granular systems in which particle fragmentation occurs or in more complex interparticle interactions including cohesion, capillarity, or contact wearing.

Particle size polydispersity represents the simplest case of "polydispersity." However, real granular media combine several sources of polydispersity such as different particle shapes, combined dry and wet contact interactions, particle density variation, etc.

Although the modeling of granular materials considering complex grain shapes has notably advanced in recent years (see, for example, Refs. $[36,37,58,59,64,65]$ ), they have only used mono or quasimonodisperse size distributions and a unique particle geometry. Additional efforts are necessary in order to investigate the grain polydispersity for nonspherical grains as well as to explore the behavior of mixtures of different particle shapes. Such granular materials have been poorly investigated to date.

Video samples of the simulations analyzed in this paper can be found in [66].

\section{ACKNOWLEDGMENTS}

This work was financially supported by a research grant awarded by the French Alternative Energies and Atomic Energy Commission (CEA).
[1] G. Chambon, J. Schmittbuhl, A. Corfdir, N. Orellana, M. Diraison, and Y. Géraud, Tectonophysics 426, 77 (2006).
[2] C. Sammis, G. King, and R. Biegel, Pure Appl. Geophys. 125, 777 (1987). 
[3] H. J. H. Brouwers and H. J. Radix, Cem. Concr. Res. 35, 2116 (2005).

[4] F. De Larrard and T. Sedran, Cem. Concr. Res. 32, 1699 (2002).

[5] T. Aste, Phys. Rev. E 53, 2571 (1996).

[6] R. Mahmoodi Baram, H. J. Herrmann, and N. Rivier, Phys. Rev. Lett. 92, 044301 (2004).

[7] C. Voivret, F. Radjaï, J.-Y. Delenne, and M. S. El Youssoufi, Phys. Rev. E 76, 021301 (2007).

[8] D.-H. Nguyen, E. Azéma, F. Radjai, and P. Sornay, Phys. Rev. E 90, 012202 (2014).

[9] D.-H. Nguyen, É. Azéma, P. Sornay, and F. Radjai, Phys. Rev. E 91, 032203 (2015).

[10] W. B. Fuller and S. E. Thompson, Trans. Am. Soc. Civ. Eng. 59, 67 (1907).

[11] J. D. Bernal and J. Mason, Nature (London) 188, 910 (1960).

[12] J. P. Troadec, A. Gervois, D. Bideau, and L. Oger, J. Phys. C 20, 993 (1987).

[13] D. Sutherland, J. Colloid Interface Sci. 60, 96 (1977).

[14] J. G. Berryman, Phys. Rev. A 27, 1053 (1983).

[15] P. Max, Nature (London) 188, 908 (1960).

[16] N. Ouchiyama, Ind. Eng. Chem. Fundam. 25, 125 (1986).

[17] A. B. Yu and N. Standish, Powder Technol. 55, 171 (1988).

[18] A. B. Yu and N. Standish, Powder Technol. 76, 113 (1993).

[19] E. J. R. Parteli, J. Schmidt, C. Blümel, K.-E. Wirth, W. Peukert, and T. Pöschel, Sci. Rep. 4, 6227 (2014).

[20] J. Dodds, J. Colloid Interface Sci. 77, 317 (1980).

[21] P. Richard, L. Oger, J. Troadec, and A. Gervois, Physica A 259, 205 (1998).

[22] H. Troadec, F. Radjai, S. Roux, and J. C. Charmet, Phys. Rev. E 66, 041305 (2002).

[23] W. Zhou, K. Xu, G. Ma, L. Yang, and X. Chang, Granular Matter 18, 1 (2016).

[24] P. S. Dodds and J. S. Weitz, Phys. Rev. E 65, 056108 (2002).

[25] H. J. Herrmann, R. Mahmoodi Baram, and M. Wackenhut, Physica A 330, 77 (2003).

[26] T. Aste and T. Di Matteo, Phys. Rev. E 77, 021309 (2008).

[27] N. Estrada and W. F. Oquendo, Phys. Rev. E 96, 042907 (2017).

[28] N. Estrada, Phys. Rev. E 94, 062903 (2016).

[29] J. Wiącek and M. Molenda, Int. J. Solids Struct. 51, 4189 (2014).

[30] J. Wiącek and M. Molenda, Granular Matter 20, 1 (2018).

[31] C. Voivret, F. Radjai, J. Y. Delenne, and M. S. El Youssoufi, Phys. Rev. Lett. 102, 178001 (2009).

[32] E. Azéma, S. Linero, N. Estrada, and A. Lizcano, Phys. Rev. E 96, 022902 (2017).

[33] J.-J. Moreau, Eur. J. Mech., A, Solids 13, 93 (1994).

[34] M. Jean, Comput. Methods Appl. Mech. Eng. 177, 235 (1999).

[35] F. Radjai and V. Richefeu, Mech. Mater. 41, 715 (2009).

[36] E. Azéma, N. Estrada, and F. Radjaï, Phys. Rev. E 86, 041301 (2012).

[37] E. Azéma, F. Radjai, and F. Dubois, Phys. Rev. E 87, 062203 (2013).
[38] E. Azéma, I. Preechawuttipong, and F. Radjaï, Phys. Rev. E 94, 042901 (2016).

[39] M. Renouf and P. Alart, Comput. Methods Appl. Mech. Eng. 194, 2019 (2005).

[40] E. Azéma, Y. Descantes, N. Roquet, J.-N. Roux, and F. Chevoir, Phys. Rev. E 86, 031303 (2012).

[41] D. Cantor, E. Azéma, P. Sornay, and F. Radjai, Comput. Particle Mech. 4, 441 (2017).

[42] J. Bardet, Experimental Soil Mechanics (Prentice Hall, Englewood Cliffs, NJ, 1997).

[43] G. D. R. Midi, Eur. Phys. J. E: Soft Matter Biol. Phys. 14, 341 (2004).

[44] P. Lacey, Chem. Eng. Res. Des. 75, S49 (1997).

[45] J. Mitchell and K. Soga, Fundamentals of Soil Behavior (Wiley, New York, 2005).

[46] B. Andreotti, Y. Forterre, and O. Pouliquen, Granular Mmedia: Between Fluid and Solid (Cambridge University Press, Cambridge, UK, 2013).

[47] I. Agnolin and J.-N. Roux, Phys. Rev. E 76, 061304 (2007).

[48] C.-H. Liu, S.-R. Nagel, D.-A. Schecter, S. Coppersmith, S. Majumdar, O. Narayan, and T.-A. Witten, Science 269, 513 (1995).

[49] D. M. Mueth, H. M. Jaeger, and S. R. Nagel, Phys. Rev. E 57, 3164 (1998).

[50] F. Radjai, M. Jean, J.-J. Moreau, and S. Roux, Phys. Rev. Lett. 77, 274 (1996).

[51] G. Løvoll, K.-J. Måløy, and E.-G. Flekkøy, Phys. Rev. E 60, 5872 (1999).

[52] S. G. Bardenhagen, J. U. Brackbill, and D. L. Sulsky, Phys. Rev. E 62, 3882 (2000).

[53] S. J. Antony, Phys. Rev. E 63, 011302 (2000).

[54] L. E. Silbert, G. S. Grest, and J. W. Landry, Phys. Rev. E 66, 061303 (2002).

[55] E. Azéma, F. Radjaï, R. Peyroux, and G. Saussine, Phys. Rev. E 76, 011301 (2007).

[56] I. Zuriguel, T. Mullin, and J. M. Rotter, Phys. Rev. Lett. 98, 028001 (2007).

[57] P. T. Metzger, Phys. Rev. E 77, 011307 (2008).

[58] E. Azéma and F. Radjaï, Phys. Rev. E 81, 051304 (2010).

[59] E. Azéma and F. Radjaï, Phys. Rev. E 85, 031303 (2012).

[60] B. Saint-Cyr, J.-Y. Delenne, C. Voivret, F. Radjai, and P. Sornay, Phys. Rev. E 84, 041302 (2011).

[61] M. Oda, J. Koshini, and S. Nemat-Nasser, Géotechnique 30, 479 (1980).

[62] N.-P. Kruyt, Mech. Mater. 44, 120 (2012).

[63] L. Rothenburg and R.-J. Bathurst, Géotechnique 39, 601 (1989).

[64] CEGEO, B. Saint-Cyr, K. Szarf, C. Voivret, E. Azéma, V. Richefeu, J.-Y. Delenne, G. Combe, C. Nouguier-Lehon, P. Villard et al., Europhys. Lett. 98, 44008 (2012).

[65] M. Boton, E. Azéma, N. Estrada, F. Radjaï, and A. Lizcano, Phys. Rev. E 87, 032206 (2013).

[66] www.cgp-gateway.org/ref039. 